Recepción: 15 / 04 / 2017

Ingeniería

Aceptación: 01 / 05 / 2017

Artículo Científico

Publicación: 15 / 05 / 2017

\title{
Diseño y construcción de un prototipo de medidor de Venturi con fines didácticos y aplicación a la ingeniería agronómica
}

\section{Design and construction of a prototype Venturi meter for teaching purposes and application engineering}

\section{Projeto e construção de um protótipo de medidor Venturi para fins de ensino e engenharia de aplicação}

Jaime A. Pazmiño-Mayorga jpazmiño@uce.edu.ec - jaipaz09@yahoo.es

Jorge S. García-Espinoza " jsgarcia@uce.edu.ec

Lazaro J. Fernández-Peinado ljfernandez@uce.edu.ec

Catalina M. Cisneros-Paredes ${ }^{\mathrm{IV}}$ cmcisneros@uce.edu.ec

Correspondencia: jpazmiño@uce.edu.ec

Ingeniero Civil; Magister en Docencia Universitaria y Administración Educativa; Docente de la Universidad Central del Ecuador.

II. Magister en Docencia Matemática; Licenciado en Ciencias de la Educación Profesor de Enseñanza media en la Especialización de Matemáticas y Física; Doctor en Ciencias de la Educación mención Gerencia Educativa; Docente Titular de la Facultad de Ingeniería, Ciencias Físicas y Matemática; Carrera de Ingeniería Civil de la Universidad Central del Ecuador.

III. Master en Gerencia de la Ciencia y la Innovación; Master of Science en Ingeniería; Ingeniero Físico; Docente Titular de la Facultad de Ingeniería, Ciencias Físicas y Matemática; Carrera de Ingeniería Civil de la Universidad Central del Ecuador.

Iv. Ingeniera Informática; Universidad Central del Ecuador. 


\section{Resumen}

Este trabajo tiene como objetivo general la elaboración de un prototipo de medidor de Venturi para aplicarlo en las clases experimentales de Hidrodinámica, con aplicación en la Educación Superior. Este prototipo permite medir el caudal o gasto de un líquido que circula por una tubería horizontal.

Con el uso de instrumentos de medición como cronómetro, probeta, calibrador y regla, se obtienen valores de tiempo y volumen que permiten calcular el caudal del fluido aplicando la definición de caudal y la ecuación de continuidad. Además con los valores observados de la diferencia de alturas de las ramas de mercurio en el manómetro se calcula el caudal aplicando el Principio de Bernoulli.

Se puede validar la eficiencia del prototipo comparando la medida del caudal aforado y el calculado con la utilización del manómetro del mercurio del prototipo. Con la materialización de este trabajo se pudo generar una nueva práctica de laboratorio orientada hacia la enseñanza de la física experimental y los resultados obtenidos se vincularán con el diseño de sistemas de riego en la agronomía.

En la construcción de este dispositivo se utilizaron materiales de bajo costo.

Palabras clave: Medidor de Venturi; hidrodinámica; teorema de Bernoulli. 


\begin{abstract}
This work has as general objective the development of a prototype Venturi meter for use in the experimental classes of Hydrodynamics, with applications at University. This prototype can measure the flow or expense of a liquid flowing through a horizontal pipe.
\end{abstract}

With the use of measuring instruments such as stopwatch, specimen, calibrator and ruler we can get time and volume values that help to calculate the fluid flow rate by applying the definition of flow and continuity equation.

In addition to the observed values of the height difference of the branches of mercury on the manometer the flow rate is calculated using the Bernoulli principle.

It can be validated by comparing the efficiency of the prototype volumetric flow measurement and calculated using this prototype. With the realization of this work could generate a new laboratory guide for teaching experimental physics and results will be linked with the design of irrigation systems in agronomy.

In the construction of this device were used cheap materials.

Key words: Venturi meter; hydrodynamics; Bernoulli principle. 


\section{Resumo}

Este trabalho tem como objetivo geral o desenvolvimento de um protótipo de medidor Venturi para uso nas classes experimentais de Hidrodinâmica, com aplicações na Universidade. Este protótipo pode medir o fluxo ou gasto de um líquido que flui através de um tubo horizontal.

Com o uso de instrumentos de medição como cronômetro, espécime, calibrador e régua, podemos obter valores de tempo e volume que ajudam a calcular o caudal de fluido aplicando a definição de equação de fluxo e continuidade.

Além dos valores observados da diferença de altura dos ramos de mercúrio no manômetro, o caudal é calculado usando o princípio de Bernoulli.

Pode ser validado através da comparação da eficiência do protótipo de medição do caudal volumétrico e calculado utilizando este protótipo. Com a realização deste trabalho poderia gerar um novo laboratório guia para o ensino de física experimental e os resultados serão associados com o projeto de sistemas de irrigação em agronomia.

Na construção deste dispositivo foram utilizados materiais baratos.

Palavras chave: Venturi metros; Hidrodinâmica; Bernoulli. 


\section{Introducción.}

La hidrodinámica es una de las ramas de la Física que más interacción tiene con la ingeniería y con los fenómenos de la naturaleza. Estudia las propiedades de los fluidos hídricos, las principales leyes de la mecánica aplicadas a los mismos y las diferentes teorías que soportan el conocimiento de esta importante rama de la ciencia. En la Universidad Central del Ecuador se estudia hidrodinámica como parte de las mallas curriculares, en la asignatura Física. (Landro \& González, 2013) (Alonso \& Rojo, 1979) (Ignacio \& Urquia, 1984)

El principio de Bernoulli, también denominado ecuación de Bernoulli o Trinomio de Bernoulli, describe el movimiento de un fluido a lo largo de un tubo de corriente. Fue expuesto por Daniel Bernoulli en su obra: Hidrodinámica (1738) y expresa que: "La energía total por unidad de volumen de un fluido incompresible animado de movimiento estacionario es constante" (fluido ideal). (Frish \& Timoreva, 1977) (Pedroza González, Ortiz Medel, \& Martínez González, 2007)

Esta energía por unidad de volumen es la suma de la variación de:

1. Energía Cinética por unidad de volumen: es la energía debida a la velocidad que posea el fluido. Conocida como presión dinámica.

$$
\frac{E c}{\Delta V}=\frac{1}{2} v^{2} \rho
$$

\section{Ecuación $N^{\circ}$ 1.- Energía Cinética por unidad de volumen.}

2. Energía Potencial Gravitacional por unidad de volumen: es la energía debido a la altitud que un fluido posee. Conocida como presión hidráulica o altimétrica. 


$$
\frac{E g}{\Delta V}=\rho g Y
$$

\section{Ecuación $N^{\circ}$ 2.- Energía Potencial Gravitacional por unidad de volumen.}

3. Energía de flujo o Energía Potencial de Presión por unidad de Volumen: es la energía que un fluido contiene debido a la presión que posee. Conocida como presión estática.

\section{$P$}

La suma de estas energías se conoce como "Ecuación de Bernoulli" (Trinomio de Bernoulli)

$$
\begin{gathered}
\frac{E c}{\Delta V}+\frac{E g}{\Delta V}+P=\text { constante } \\
\frac{1}{2} v^{2} \rho+\rho g Y+P=\text { constante }
\end{gathered}
$$

Ecuación $\mathbf{N}^{\circ}$ 3: Ecuación de Bernoulli o Conservación de la energía del fluido.

Dónde:

$v$ Velocidad del fluido en la sección considerada

$\rho$ Densidad del fluido

$P \quad$ Presión a lo largo de la línea de corriente

$g$ Aceleración gravitatoria

$Y \quad$ Altura en la dirección de la gravedad desde una cota de referencia

La ecuación 3 representa la conservación de la energía del fluido usada en fluidos ideales y su uso es limitado para fluidos compresibles como los gases. 
En el movimiento de un fluido líquido por una tubería de sección variable se deduce que en los lugares de menor área de la tubería aumente la velocidad del fluido, disminuyendo la presión estática, caso contrario en los lugares de mayor área.

\section{Dispositivo de Venturi}

Este dispositivo se utiliza en oxigenoterapia, medición del caudal de agua en el suministro para uso doméstico o industrial, en los sistemas de riego, entre otros; los cuales están basados en el principio de Bernoulli. Ver Figura $N^{\circ} 1$.

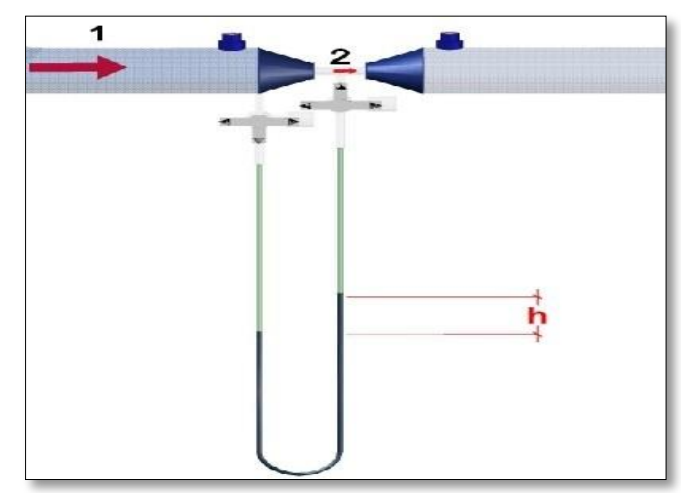

Figura $N^{\circ}$ 1.- Dispositivo de Venturi en tubería horizontal.

Diseño y disposición final del Prototipo del Medidor de Venturi

A continuación, se puede observar el diseño y el prototipo construido y listo para su funcionamiento. Ver Figura $N^{\circ} 2$ y Figura $N^{\circ} 3$. 

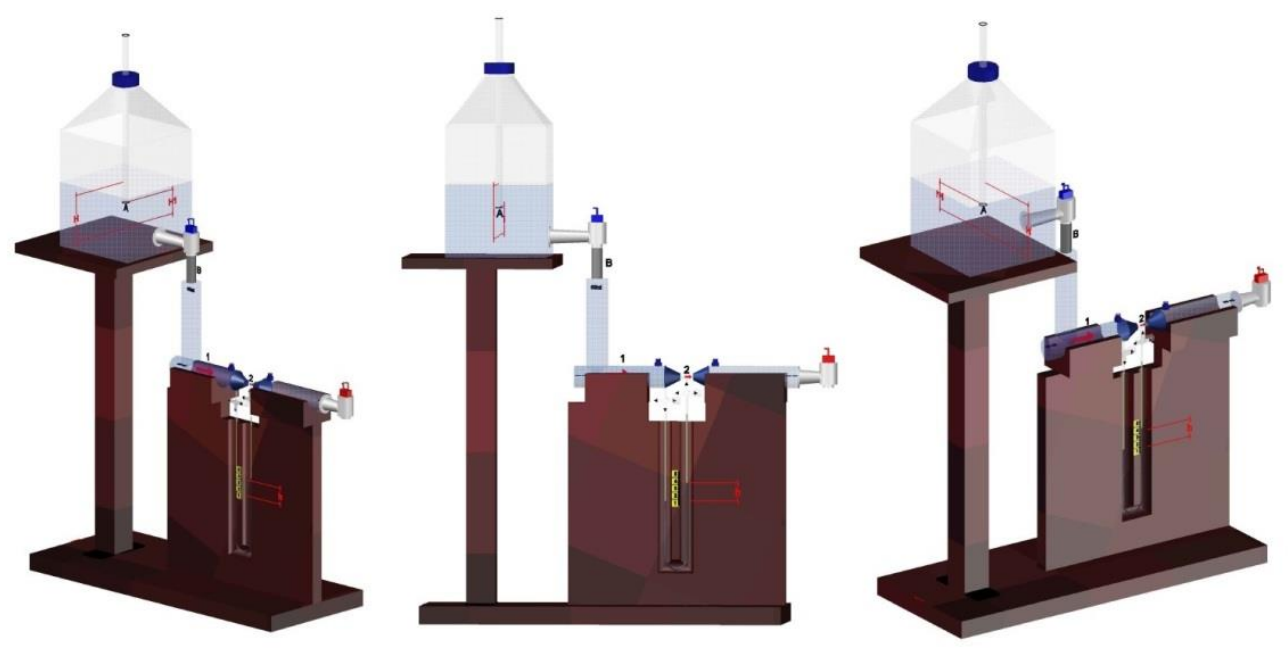

Figura $N^{\circ}$ 2.- Diseño Prototipo del Medidor de Venturi.

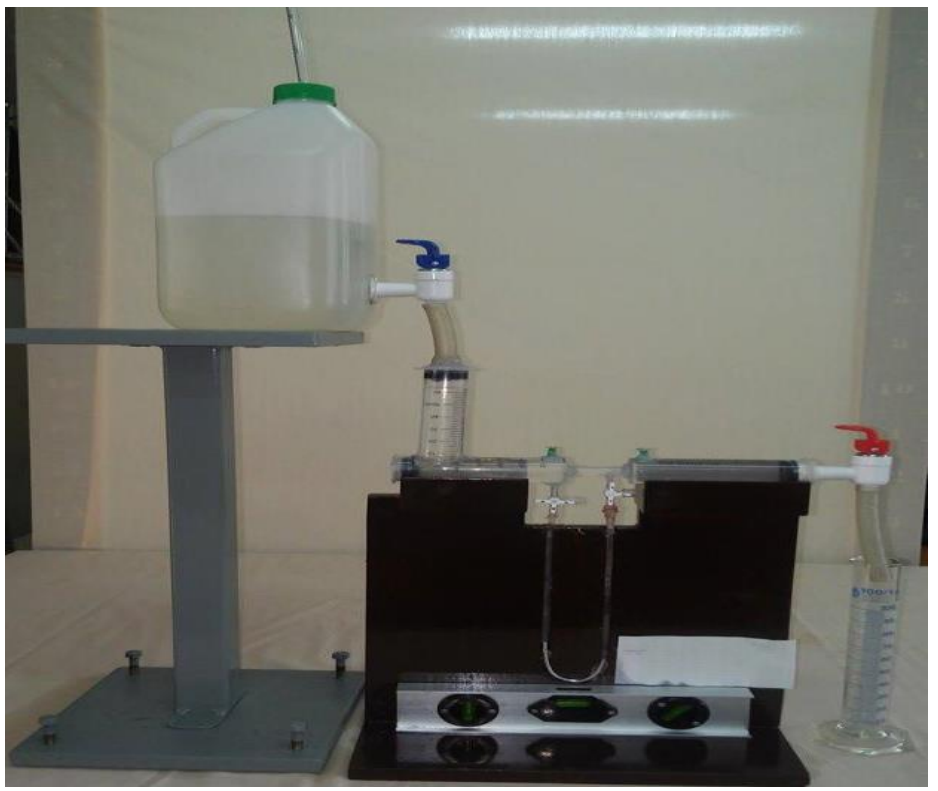

Figura $N^{\circ}$ 3.- Prototipo del Medidor de Venturi. 


\section{Aplicaciones del Principio de Bernoulli}

\section{Chimenea}

Las chimeneas son tubos muy largos para alcanzar grandes alturas en donde la velocidad del viento tiende a ser constante y elevada. Cuanto más rápidamente sopla el viento sobre la boca de una chimenea, más baja es la presión y mayor es la diferencia de presión entre la base y la boca de la chimenea, en consecuencia, los gases de combustión se extraen mejor.

\section{Tubería}

La ecuación de Bernoulli y la ecuación de continuidad también indican que, si reducimos el área transversal de una tubería para que aumente la velocidad del fluido que pasa por ella, se reducirá la presión.

\section{Natación}

La aplicación dentro de este deporte se ve reflejada directamente cuando las manos del nadador cortan el agua generando una menor presión y mayor propulsión.

\section{Carburador}

En un carburador de automóvil, la presión del aire que pasa a través del cuerpo del carburador, disminuye cuando pasa por un estrangulamiento. Al disminuir la presión, la gasolina fluye, se vaporiza y se mezcla con la corriente de aire. 


\section{Aireadores}

Una de las aplicaciones de la ecuación de Bernoulli es en la construcción de los denominados aireadores. La aireación es el proceso mediante el cual se puede mezclar, circular, o disolver aire dentro de un líquido u otra sustancia, lo que permite realizar el tratamiento de aguas residuales y su posterior utilización en la Ingeniería Agronómica.

\section{Ingeniería Agronómica}

Se utiliza este dispositivo para medir la velocidad y el caudal de un río, que a su vez es un parámetro de diseño para un canal o el desvío del agua para riego por gravedad.

\section{Método.}

Con el uso del método investigativo experimental se determinó como variables independientes: volumen, tiempo y altura, dependiente el caudal; en función de que son parámetros observables y medibles experimentalmente. Considerando que volumen, tiempo y altura se miden directamente, en cambio el caudal es resultado de una medición indirecta en función de las anteriores.

\section{Materiales}

Los materiales utilizados para el diseño y construcción del prototipo experimental del medidor Venturi se detallan a continuación con su cantidad, precio unitario y precio total. 


\begin{tabular}{|l|c|cc|c|}
\hline Materiales & Cantidad & $\begin{array}{l}\text { Unidad } \\
\text { medida }\end{array}$ & $\begin{array}{l}\text { de Precio } \\
\text { Unitario }\end{array}$ & $\begin{array}{l}\text { Precio } \\
\text { Total }\end{array}$ \\
\hline Base de madera & 2 & $\mathrm{U}$ & 3 & 6 \\
\hline Tubo metálico & 2 & $\mathrm{U}$ & 2 & 4 \\
\hline $\begin{array}{l}\text { Pegamento líquido extra } \\
\text { fuerte }\end{array}$ & 1 & $\mathrm{U}$ & 1,5 & 1,5 \\
\hline Envase Plástico de 5 1 & 1 & $\mathrm{U}$ & 2 & 2 \\
\hline Equipo de venoclisis & 1 & $\mathrm{U}$ & 1 & 1 \\
\hline Jeringa 10 ml & 1 & $\mathrm{U}$ & 0,3 & 0,3 \\
\hline Jeringa 60 ml & 3 & $\mathrm{U}$ & 1 & 3 \\
\hline Llave de dispensador & 2 & $\mathrm{U}$ & 1 & 2 \\
\hline $\begin{array}{l}\text { Manguera de caucho 0,013 } \\
\text { m }\end{array}$ & 1 & $\mathrm{~m}$ & 1 & 1 \\
\hline Mercurio líquido & 25 & $\mathrm{~g}$ & 1 & 25 \\
\hline Barras de silicona & 15 & $\mathrm{U}$ & 0,2 & 3 \\
\hline Tubo de silicona líquida & 3 & $\mathrm{U}$ & 2 & 6 \\
\hline Tubo de vidrio delgado & 1 & $\mathrm{U}$ & 4 & 4 \\
\hline $\begin{array}{l}\text { Válvula de suero quirúrgico } \\
\text { de 3 salidas }\end{array}$ & 2 & $\mathrm{U}$ & 1 & 2 \\
\hline Probeta graduada de 500 ml & 1 & $\mathrm{U}$ & 14 & 14 \\
\hline & & $\mathrm{TOTAL} \mathrm{USD}$ & 74,8 \\
\hline
\end{tabular}

Tabla $N^{\circ} 1$. Gastos y materiales del prototipo de Medidor de Venturi.

*Precios de Ecuador 


\section{Resultados y discusión.}

En la Unidad de Física de la Universidad Central del Ecuador se pudo determinar una necesidad en las prácticas relacionadas con el tema de la Hidromecánica. Para este efecto se planificó la creación de un prototipo que permita comprobar las ecuaciones de continuidad y el Teorema de Bernoulli. Con los estudiantes se organizaron equipos de trabajo para el diseño y construcción de algunos modelos de medidor de Venturi, seleccionando los que más se ajustan a los objetivos propuestos, los cuales se sometieron a un proceso de mejora en cuanto se refiere a materiales, construcción, fundamentación teórico - práctica y a la obtención de resultados. Luego de un proceso de experimentación se fueron corrigiendo cada uno de los aspectos mencionados, de tal forma que con el modelo experimental obtenido se lograron mejores resultados en la comprobación del Teorema de Bernoulli. (Halliday, Resnick, \& Krane, 1999) (Bueche, 2006)

Se probaron diferentes líquidos no miscibles para utilizarlo como líquido manométrico: agua, glicerina, clara de huevo, miel de abeja diluida, gasolina, diesel, entre otros; concluyendo en que el más idóneo es el mercurio. Para lograr la estabilización del flujo del líquido, se eliminaron factores que inciden en el aumento del porcentaje de error, como los flujos variables y el denominado golpe de ariete provocado por la manipulación de válvulas reguladoras.

Medición experimental del caudal que fluye en los tubos horizontales:

Medición 1: Con los valores del tiempo y volumen, utilizando instrumentos como el cronómetro y probeta graduada (definición de caudal).

Medición 2: Con los valores de los diámetros de la tubería y la diferencia de alturas de la columna de mercurio en el manómetro del medidor de Venturi (Teorema de Bernoulli). 
Se compararon los resultados obteniendo el margen de error que no sobrepasa el $2 \%$, válido para este tipo de experimento donde intervienen factores de apreciación humana.

¿Cómo se eliminaron los flujos variables?

\section{Frasco de Mariotte}

En la elaboración del prototipo experimental debíamos contar con un gasto o caudal constante de agua, por lo que se decidió acoplar al medidor de Venturi el Frasco de Mariotte, como se puede observar en la siguiente Figura $N^{\circ} 4$.

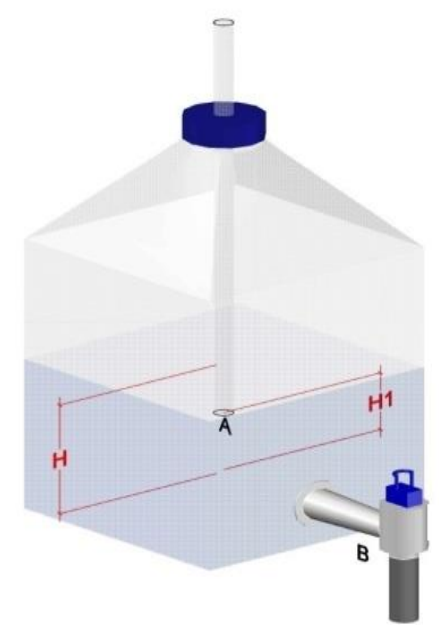

Figura $N^{\circ}$ 4.- Frasco de Mariotte.

Este dispositivo consiste en un recipiente con una perforación en la parte inferior para la salida del líquido (Posición B). La parte superior del recipiente tiene un tapón con un tubo delgado de vidrio abierto en sus dos extremos, insertado de manera que el extremo superior esté fuera del recipiente y, atravesando el tapón, el extremo inferior se sumerge en el líquido (Posición A). 
En el instante en que sale el líquido (Posición B), el nivel en la parte superior del frasco inicia su descenso (Posición H), haciendo que la cantidad de aire contenida sobre el líquido aumente su volumen y disminuya la densidad; esto permite que el líquido dentro del tubo delgado descienda hasta que ingresa aire en forma de burbujas, haciendo que la presión dentro del frasco se equilibre con la presión atmosférica, este fenómeno se mantiene hasta que el líquido alcance el nivel inferior del tubo (Posición A), momento para el cual la velocidad de salida del fluido (Posición B) dependerá de la altura de la parte inferior del tubo (Posición $\mathrm{H}_{1}$ ) en donde la velocidad inicia su disminución. Siempre que el líquido dentro del frasco se encuentre por encima del extremo inferior del tubo de vidrio, se logra que el caudal sea constante, de acuerdo al Teorema de Bernoulli.

La posición A interno y B externo de salida del líquido están en contacto con la atmósfera por tanto están sometidos a la presión atmosférica; el nivel de referencia se ubica en la salida del líquido (Posición B), donde $\mathrm{Y}_{B}=0$ y $\mathrm{Y}_{A}=H_{1}$.

La velocidad constante de salida a través del orificio, posición B, se deduce de la aplicación de la ecuación de Bernoulli entre los puntos A y B, considerando que la velocidad inicial en la posición A es nula y que en este punto actúa directamente la presión atmosférica, de esta forma la ecuación es:

$$
\begin{gathered}
\frac{E c_{A}}{\Delta V}+\frac{E g_{A}}{\Delta V}+P_{A}=\frac{E c_{B}}{\Delta V}+\frac{E g_{B}}{\Delta V}+P_{B} \\
\frac{1}{2} \rho_{H_{2} O}\left(\mathrm{~V}_{\mathrm{A}}\right)^{2}+\rho_{\mathrm{H}_{2} O} * \mathrm{~g} * \mathrm{Y}_{\mathrm{A}}+\mathrm{P}_{\mathrm{A}}=\frac{1}{2} \rho_{\mathrm{H}_{2} O}\left(\mathrm{~V}_{\mathrm{B}}\right)^{2}+\rho_{\mathrm{H}_{2} O} * \mathrm{~g} * \mathrm{Y}_{B}+\mathrm{P}_{\mathrm{B}}
\end{gathered}
$$

Ecuación $N^{\circ}$ 4.- Ecuación de Bernoulli en la posición A y B. 


\section{Dónde:}

$P_{A}$ y $P_{B} \quad$ Presión atmosférica en la posición A y B.

$V_{A}$ y $V_{B} \quad$ Velocidad en la posición A y B.

$Y_{A}$ y $Y_{B} \quad$ Altura de la posición A y B.

$\rho \quad$ Densidad del fluido (agua).

$g \quad$ Aceleración gravitatoria.

Bajo las siguientes condiciones:

$$
\begin{aligned}
\mathrm{P}_{\mathrm{A}} & =\mathrm{P}_{\mathrm{B}} \\
V_{A} & =0 \frac{\mathrm{m}}{\mathrm{s}} \\
Y_{A} & =H_{1} \\
Y_{B} & =0 \mathrm{~m}
\end{aligned}
$$

Por tanto:

$$
\begin{aligned}
& \frac{1}{2} \rho_{\mathrm{H}_{2} \mathrm{O}}\left(\mathrm{V}_{\mathrm{A}}\right)^{2}+\rho * \mathrm{~g} * \mathrm{Y}_{\mathrm{A}}=\frac{1}{2} \rho_{\mathrm{H}_{2} \mathrm{O}}\left(\mathrm{V}_{\mathrm{B}}\right)^{2}+\rho_{\mathrm{H}_{2} \mathrm{O}} * \mathrm{~g} * \mathrm{Y}_{B} \\
& \frac{1}{2} \rho_{\mathrm{H}_{2} \mathrm{O}}(0)^{2}+\rho_{\mathrm{H}_{2} \mathrm{O}} * \mathrm{~g} * \mathrm{H}_{1}=\frac{1}{2} \rho_{\mathrm{H}_{2} \mathrm{O}}\left(\mathrm{V}_{\mathrm{B}}\right)^{2}+\rho_{\mathrm{H}_{2} \mathrm{O}} * \mathrm{~g} *(0) \\
& \rho_{\mathrm{H}_{2} \mathrm{O}} * \mathrm{~g} * \mathrm{H}_{1}=\frac{1}{2} \rho_{\mathrm{H}_{2} \mathrm{O}}\left(\mathrm{V}_{\mathrm{B}}\right)^{2} \\
& 2 * \mathrm{~g} * \mathrm{H}_{1}=\mathrm{V}_{B}^{2} \\
& \mathrm{~V}_{B}=\sqrt{2 * \mathrm{~g} * \mathrm{H}_{1}}
\end{aligned}
$$

Ecuación $N^{\circ}$ 5.- Ecuación de la velocidad en la posición $B$ 
Esta velocidad de salida nos garantiza un caudal constante que fluye en el tubo de Bernoulli.

¿Qué es el golpe de ariete?

El golpe de ariete o choque hidráulico ocurre cuando varía bruscamente la presión de un fluido dentro de una tubería, motivado por el cierre o abertura de una llave, grifo o válvula; también puede producirse por la puesta en marcha o detención de un motor o bomba hidráulica.

¿Cómo se eliminó el efecto de golpe de ariete?

En la medición del caudal utilizando la definición es necesario medir el tiempo para un volumen determinado, por tanto para eliminar el referido golpe de ariete, se hizo necesario desechar el volumen comprendido entre los valores $(0-100) \mathrm{ml}$, en la probeta, y tener como punto de referencia los $100 \mathrm{ml}$.

Registro de valores experimentales

\section{Medición 1:}

Para medir el caudal de líquido abrimos la llave del frasco de Mariotte y la llave de salida del medidor de Venturi donde se coloca la probeta graduada, dejamos que fluya el agua por el prototipo y, con el cronómetro previamente encerado, medimos el tiempo que tarda en la probeta lograr volúmenes de $100 \mathrm{ml}, 200 \mathrm{ml}, 300 \mathrm{ml} \mathrm{y} 400 \mathrm{ml}$; a partir del nivel de referencia en los $100 \mathrm{ml}$ iniciales en los que el flujo de líquido se estabiliza evitando el golpe de ariete o pulso de Zhukowski.

Los resultados obtenidos del volumen de líquido y sus tiempos respectivos se expresan en la Tabla $N^{\circ} 2$. 
Los caudales individuales son calculados usando:

$$
Q_{i}=\frac{V_{i}}{t_{p i}} ; \quad \text { Para } \mathrm{i}=1,2,3,4
$$

\section{Ecuación $N^{\circ}$ 6.- Ecuación de la definición de caudal}

Dónde:

$V_{i} \quad$ Volumen del fluido en la probeta graduada

$t_{p i}$ Tiempo promedio en que demora el fluido en llegar al volumen solicitado

$Q_{i}$ Caudal para cada volumen tomado

$$
Q_{P}=\frac{\sum Q_{i}}{n}=\frac{Q_{1}+Q_{2}+Q_{3}+Q_{4}}{4}
$$

\section{Ecuación $N^{\circ}$ 7.- Ecuación del caudal promedio}

Dónde:

$Q_{p 1}$ Caudal promedio por definición 
Jaime A. Pazmiño-Mayorga; Jorge S. García-Espinoza; Lazaro J. Fernández-Peinado; Catalina M. Cisneros-Paredes

\begin{tabular}{|c|c|c|c|c|c|c|c|c|}
\hline $\begin{array}{l}\text { Volumen } \\
\left(10^{-6} \mathrm{~m}^{3}\right)\end{array}$ & $\mathrm{t}_{1}(\mathrm{~s})$ & $\mathrm{t}_{2}(\mathrm{~s})$ & $\mathrm{t}_{3}(\mathrm{~s})$ & $\mathrm{t}_{4}(\mathrm{~s})$ & $t_{5}(s)$ & $t_{p}(s)$ & $\left(10^{-6} \mathrm{~m}^{3} / \mathrm{s}\right)$ & $\begin{array}{c}\mathrm{Q}_{\mathrm{p} 1} \\
\left(10^{-6} \mathrm{~m}^{3} / \mathrm{s}\right)\end{array}$ \\
\hline 100 & 2,31 & 2,18 & 2,21 & 2,26 & 2,22 & 2,24 & 44,72 & \multirow{4}{*}{44,47} \\
\hline 200 & 4,51 & 4,38 & 4,46 & 4,38 & 4,32 & 4,41 & 45,35 & \\
\hline 300 & 6,75 & 6,78 & 6,93 & 6,77 & 6,68 & 6,78 & 44,23 & \\
\hline 400 & 9,06 & 9,25 & 9,46 & 9,20 & 8,94 & 9,18 & 43,56 & \\
\hline
\end{tabular}

\section{Tabla $N^{\circ}$ 2.- Medidor de Venturi - Caudal usando volumen y tiempo}

Medición 2:

Para el movimiento del fluido en la tubería de eje horizontal, designando con 1 la sección ancha y con 2 la sección angosta tenemos, según el Teorema de Bernoulli:

$$
\begin{gathered}
\frac{E c_{1}}{\Delta V}+\frac{E g_{1}}{\Delta V}+P_{1}=\frac{E c_{2}}{\Delta V}+\frac{E g_{2}}{\Delta V}+P_{2} \\
\frac{1}{2} \rho\left(\mathrm{V}_{1}\right)^{2}+\rho_{H_{2} O} * g * \mathrm{Y}_{1}+\mathrm{P}_{1}=\frac{1}{2} \rho\left(\mathrm{V}_{2}\right)^{2}+\rho_{H_{2} O} * \mathrm{~g} * \mathrm{Y}_{2}+\mathrm{P}_{2}
\end{gathered}
$$

Como: $\mathrm{Y}_{1}=Y_{2}$

$$
\begin{gathered}
\mathrm{P}_{1}+\frac{1}{2} \rho_{\mathrm{H}_{2} O}\left(\mathrm{~V}_{1}\right)^{2}=\mathrm{P}_{2}+\frac{1}{2} \rho_{\mathrm{H}_{2} O}\left(\mathrm{~V}_{2}\right)^{2} \\
\mathrm{P}_{1}-\mathrm{P}_{2}=\frac{1}{2} \rho_{\mathrm{H}_{2} \mathrm{O}}\left[\left(\mathrm{V}_{2}\right)^{2}-\left(\mathrm{V}_{1}\right)^{2}\right] \\
\frac{2\left(\mathrm{P}_{1}-\mathrm{P}_{2}\right)}{\rho_{\mathrm{H}_{2} O}}=\left(\mathrm{V}_{2}\right)^{2}-\left(\mathrm{V}_{1}\right)^{2}
\end{gathered}
$$


Por la ecuación de la continuidad se tiene:

$$
\mathrm{A}_{1} V_{1}=\mathrm{A}_{2} V_{2}
$$

\section{Ecuación $N^{\circ}$ 8.- Ecuación de continuidad}

$$
V_{2}=\frac{A_{1}}{A_{2}} V_{1}
$$

Reemplazando:

$$
\begin{gathered}
\frac{2\left(\mathrm{P}_{1}-\mathrm{P}_{2}\right)}{\rho_{\mathrm{H}_{2} O}}=\left(\frac{\mathrm{A}_{1}}{\mathrm{~A}_{2}} \mathrm{~V}_{1}\right)^{2}-\left(\mathrm{V}_{1}\right)^{2} \\
\frac{2\left(\mathrm{P}_{1}-\mathrm{P}_{2}\right)}{\rho_{\mathrm{H}_{2} O}}=\mathrm{V}_{1}^{2}\left(\frac{\mathrm{A}_{1}^{2}-A_{2}^{2}}{\mathrm{~A}_{2}^{2}}\right) \\
\frac{2\left(\mathrm{P}_{1}-\mathrm{P}_{2}\right) \mathrm{A}_{2}^{2}}{\rho_{\mathrm{H}_{2} O}\left(\mathrm{~A}_{1}^{2}-A_{2}^{2}\right)}=\mathrm{V}_{1}^{2} \\
\mathrm{~V}_{1}=A_{2} \sqrt{\frac{2\left(\mathrm{P}_{1}-\mathrm{P}_{2}\right)}{\rho_{H_{2} O}\left(\mathrm{~A}_{1}^{2}-A_{2}^{2}\right)}} \\
\mathrm{V}_{1}=A_{2} \sqrt{\frac{2 \Delta \mathrm{P}}{\rho_{H_{2} O}\left(\mathrm{~A}_{1}^{2}-A_{2}^{2}\right)}}
\end{gathered}
$$

Ecuación $N^{\circ}$ 9.- Ecuación de la velocidad en la posición 1 
Para la sección menor se tiene:

$$
V_{2}=A_{1} \cdot \sqrt{\frac{2 . \Delta P}{\rho_{H_{2} O}\left(A_{1}^{2}-A_{2}^{2}\right)}}
$$

\section{Ecuación $N^{\circ} 10 .-$ Ecuación de la velocidad en la posición 2}

Dónde:

$\Delta P \quad$ Diferencia de Presión

$\rho_{\mathrm{H}_{2} \mathrm{O}} \quad$ Densidad del fluido (agua)

$A_{1} \quad$ Área en la sección mayor

$\mathrm{A}_{2} \quad$ Área en la sección menor

$V_{1} \quad$ Velocidad del Fluido en la sección mayor

$V_{2} \quad$ Velocidad del Fluido en la sección menor

Calculando el caudal, tanto para la sección mayor como para la sección menor del tubo de eje horizontal,

$$
\begin{gathered}
Q_{1}=V_{1} \cdot A_{1} ; Q_{2}=V_{2} \cdot A_{2} \\
Q_{1}=Q_{2}
\end{gathered}
$$

\section{Dónde:}

$Q_{1} \quad$ Caudal en la sección mayor

$\mathrm{Q}_{2} \quad$ Caudal en la sección menor 
Cuando el líquido fluye por el prototipo, el manómetro colocado entre la tubería horizontal de sección mayor y menor marca una diferencia de alturas del mercurio en sus ramas, valores que se registran en la Tabla $\mathrm{N}^{\circ}$ 3. La condición hidrostática para las ramas del manómetro del mercurio está dada por la ecuación:

$$
\Delta P=g \Delta h\left(\rho^{\prime}-\rho\right)
$$

\section{Ecuación $N^{\circ}$ 11.- Ecuación de diferencia de presión en el manómetro}

Dónde:

$\Delta P \quad$ Diferencia de presión en el manómetro.

$g$ Aceleración gravitatoria.

$\Delta h \quad$ Diferencia de alturas

$\rho \quad$ Densidad del agua; $\rho=10^{3} \mathrm{~kg} / \mathrm{m}^{3}$

$\rho^{\prime} \quad$ Densidad del mercurio; $\rho^{\prime}=13,6 * 10^{3} \mathrm{~kg} / \mathrm{m}^{3}$

\begin{tabular}{|c|c|c|c|c|c|c|c|c|}
\hline & D & $\mathbf{A}$ & $\mathbf{A}^{2}$ & $\Delta \mathbf{h}$ & $\Delta \mathbf{P}$ & $\mathbf{V}$ & $Q_{i}$ & $Q_{p_{2}}$ \\
\hline Tubo & $10^{-3} \mathrm{~m}$ & $10^{-3} m^{2}$ & $10^{-9} m^{4}$ & $10^{-3} \mathrm{~m}$ & $P a$ & $\mathrm{~m} / \mathrm{s}$ & $10^{-6} \frac{\mathrm{m}^{3}}{\mathrm{~s}}$ & $10^{-6} \frac{m^{3}}{s}$ \\
\hline Mayor & 29,50 & 0,68 & 470,00 & \multirow{2}{*}{2,50} & \multirow{2}{*}{308,07} & 0,06 & 43,64 & \multirow{2}{*}{43,64} \\
\hline Menor & 8,40 & 0,05 & 3,00 & & & 0,79 & 43,64 & \\
\hline
\end{tabular}

Tabla $N^{\circ}$ 3.- Medidor de Venturi - Caudal usando el manómetro 
Dónde:

D Diámetro Tubo
A Área
V Velocidad
$Q_{p_{2}}$ Caudal promedio por Teorema de Bernoulli

Cálculo del porcentaje de error en el experimento

Caudal o gasto en el tubo horizontal por la definición

$$
Q_{p 1}=44,47 \times 10^{.6} \frac{\mathrm{m}^{3}}{\mathrm{~s}}
$$

Caudal o gasto utilizando el Teorema de Bernoulli

$$
Q_{p 2}=43,64 \times 10^{\cdot 6} \frac{\mathrm{m}^{3}}{\mathrm{~s}}
$$

Con estos valores que obtuvimos podemos realizar el cálculo del error:

$$
E=100 \%-\left(\frac{Q_{p 2}}{Q_{p 1}}\right) \times 100 \%
$$

Ecuación $N^{\circ}$ 12.- Porcentaje de error en el caudal

$$
\begin{gathered}
E=100 \%-\left(\frac{44,47 \times 10^{\cdot 6}}{43,64 \times 10^{\cdot 6}}\right) \times 100 \% \\
E=1,87 \%
\end{gathered}
$$




\section{Conclusiones.}

La construcción del prototipo de medidor de Venturi con fines didácticos es viable y representa un bajo costo, pues se emplean materiales de fácil adquisición.

Con el prototipo podemos lograr una participación activa en el uso del mismo ya que facilita la medición de los parámetros involucrados.

Al procesar los datos se constató que en el prototipo de medidor Venturi se comprueba la validez de la ecuación para medir la velocidad de un líquido que se desplaza en una tubería horizontal.

Los valores obtenidos en las mediciones 1 y 2, aportaron resultados satisfactorios dando un porcentaje de error de $1,87 \%$, que valida la sinergia de la teoría de Bernoulli con la experimentación.

La aplicación directa de este prototipo experimental en la Ingeniería es para la observación y cálculo del número de Reynols que determina si el flujo de este líquido que circula por el tubo de Venturi creado es laminar o turbulento.

La aplicación directa en la Ingeniería Agronómica es que este prototipo puede servir de base para la determinación experimental de pérdidas de carga locales y de esta manera relacionar este conocimiento con el diseño de sistemas de riego en los cuales se deben considerar este tipo de pérdidas de energía debido fundamentalmente a la utilización de accesorios en las tuberías. 


\section{Recomendaciones.}

Utilizar el prototipo de medidor de Venturi construido y validado en esta investigación para la enseñanza de la Hidrodinámica.

Organizar nuevas prácticas de laboratorio en la Unidad de Física de la Universidad Central del Ecuador

Encomendar a las autoridades de la Universidad Central del Ecuador la asignación de recursos necesarios para la réplica de este prototipo.

\section{Agradecimiento.}

Se agradece el apoyo de la Unidad de Física de la Universidad Central del Ecuador, a los compañeros Docentes e Instructores de física y a los Estudiantes de las Facultades de Ciencias Agrícolas e Ingeniería, Ciencias Físicas y Matemática.

\section{Bibliografía.}

Alonso, M., \& Rojo, O. (1979). Física Mecánica y Termodinámica. México: Addison-Wesley Iberoamericana.

Bueche, F. (2006). Física General (10 ed.). México: Mc Graw Hill.

Frish, S., \& Timoreva, A. (1977). Curso de Física General. Moscú: Mir.

Halliday, D., Resnick, R., \& Krane, K. (1999). Fisica. México: Comañia Editorial Continental .

Ignacio, J., \& Urquia, L. (1984). Energía hidráulica y eólica práctica. Navarra: Pamiela.

Landro, A., \& González, M. (2013). Bernoulli, de moivre, bayes, price y los fundamentos de la inferencia inductiva. Cuadernos del CIMBAGE, 15(1), 33-56.

Pedroza González, E., Ortiz Medel, J., \& Martínez González, F. (2007). Historia del Teorema de Bernoulli. Acta Universitaria, 17(1), 39-45. 